\title{
3D ultrasound imaging performance of a row-column addressed 2D array transducer: a simulation study
}

Rasmussen, Morten Fischer; Jensen, Jørgen Arendt

Published in:

Proceedings of SPIE

Link to article, DOI:

$10.1117 / 12.2007083$

Publication date:

2013

Link back to DTU Orbit

Citation (APA):

Rasmussen, M. F., \& Jensen, J. A. (2013). 3D ultrasound imaging performance of a row-column addressed 2D array transducer: a simulation study. In Proceedings of SPIE: Medical Imaging 2013: Ultrasonic Imaging, Tomography, and Therapy (Vol. 8675). [86750C] SPIE - International Society for Optical Engineering. https://doi.org/10.1117/12.2007083

\section{General rights}

Copyright and moral rights for the publications made accessible in the public portal are retained by the authors and/or other copyright owners and it is a condition of accessing publications that users recognise and abide by the legal requirements associated with these rights.

- Users may download and print one copy of any publication from the public portal for the purpose of private study or research.

- You may not further distribute the material or use it for any profit-making activity or commercial gain

- You may freely distribute the URL identifying the publication in the public portal 


\title{
3D ultrasound imaging performance of a row-column addressed 2D array transducer: a simulation study
}

\author{
Morten Fischer Rasmussen and Jørgen Arendt Jensen \\ Center for Fast Ultrasound Imaging, Dept. of Electrical Engineering \\ Technical University of Denmark, 2800 Lyngby, Denmark.
}

\begin{abstract}
This paper compares the imaging performance of a $128+128$ element row-column addressed array with a fully addressed $16 \times 162 \mathrm{D}$ array. The comparison is made via simulations of the point spread function with Field II. Both arrays have lambda-pitch, a center frequency of $3.5 \mathrm{MHz}$ and use 256 active elements. The row-column addressed array uses 128 transmit channels and 128 receive channels, whereas the fully addressed array uses 256 channels in both transmit and receive. The large size of the emulated row and column elements in the row-column addressed array causes ghost echoes to appear. The ghost echoes are shown to be suppressed when the sub-elements within each of the emulated row and column elements are apodized. The maximum ghost intensity is suppressed by $22.2 \mathrm{~dB}$ compared to using no apodization. With apodization applied, the full-width-at-half-maximum in the lateral direction for the fully addressed array is $2.81 \mathrm{~mm}$, and $1.01 \mathrm{~mm}$ for the row-column addressed array. This shows that the detail resolution can be more than doubled using the row-column addressed array instead of the fully addressed array. The row column addressed array achieves a $R_{20 \mathrm{~dB}}$ cystic resolution of $0.76 \mathrm{~mm}$, compared to $3.16 \mathrm{~mm}$ for the fully addressed array. The significantly smaller $R_{20 \mathrm{~dB}}$-value for the row-column addressed array indicates that it can achieve a much higher contrast resolution than the fully addressed array.
\end{abstract}

Keywords: row-column addressing, beamforming, low channel count 3D imaging, 2D array transducer, synthetic aperture imaging, medical ultrasound

\section{INTRODUCTION}

For ultrasonic 3D imaging, 2D array transducers are needed to achieve real-time scanning of a volume. ${ }^{1,2}$ The number of elements in a fully addressed $N \times N$ 2D array scales with $N^{2}$. In 2D imaging, a 1D array using more than 100 elements is often used. Using a fully addressed 2D array, this would correspond to an array with more than 10000 elements. To control the individual elements in the array, a connection has to be made to each element. Hereby, any delay or apodization scheme can be applied, offering maximum control and flexibility in the image processing. ${ }^{2-4}$ However, addressing each element individually results in a vast amount of interconnections and offers a great challenge in acquiring and processing the large amount of data. Reducing the number of transducer elements by using sparse arrays has therefore seen a great amount of interest in the last couple of decades. ${ }^{5-9}$ One of the drawbacks of sparse arrays is the lower emitted energy from the reduced number of elements. This leads to a lower SNR in the recorded ultrasound image. The sparse arrays also have higher side-lobes and can introduce grating lobes in the field.

2 D row-column addressed arrays has recently attracted some attention. ${ }^{10-13}$ In a row-column addressed array, the are elements accessed by their row index in transmit and by their column index in receive, or vice versa. This is illustrated in Fig. 1. Thus, instead of addressing a single element, an entire row or column of elements is addressed. This effectively reduces the number of elements on a $N \times N 2 \mathrm{D}$ array from $N^{2}$ to $2 N$. The new line elements, shown in the bottom left and bottom right array in Fig. 1, are in this work referred to as row and column elements. The individual physical elements, making up the row and column elements, are referred to as sub-elements or physical elements.

The purpose of this paper is to investigate the imaging performance of row-column addressed arrays. This is done by simulating the point spread function (PSF) and measuring the main lobe width and the cystic resolution. It is shown how the focusing can be achieved with a row-column addressed array. The large emulated elements in

Further author information: Send correspondence to M. F. Rasmussen. E-mail: mofi@elektro.dtu.dk 


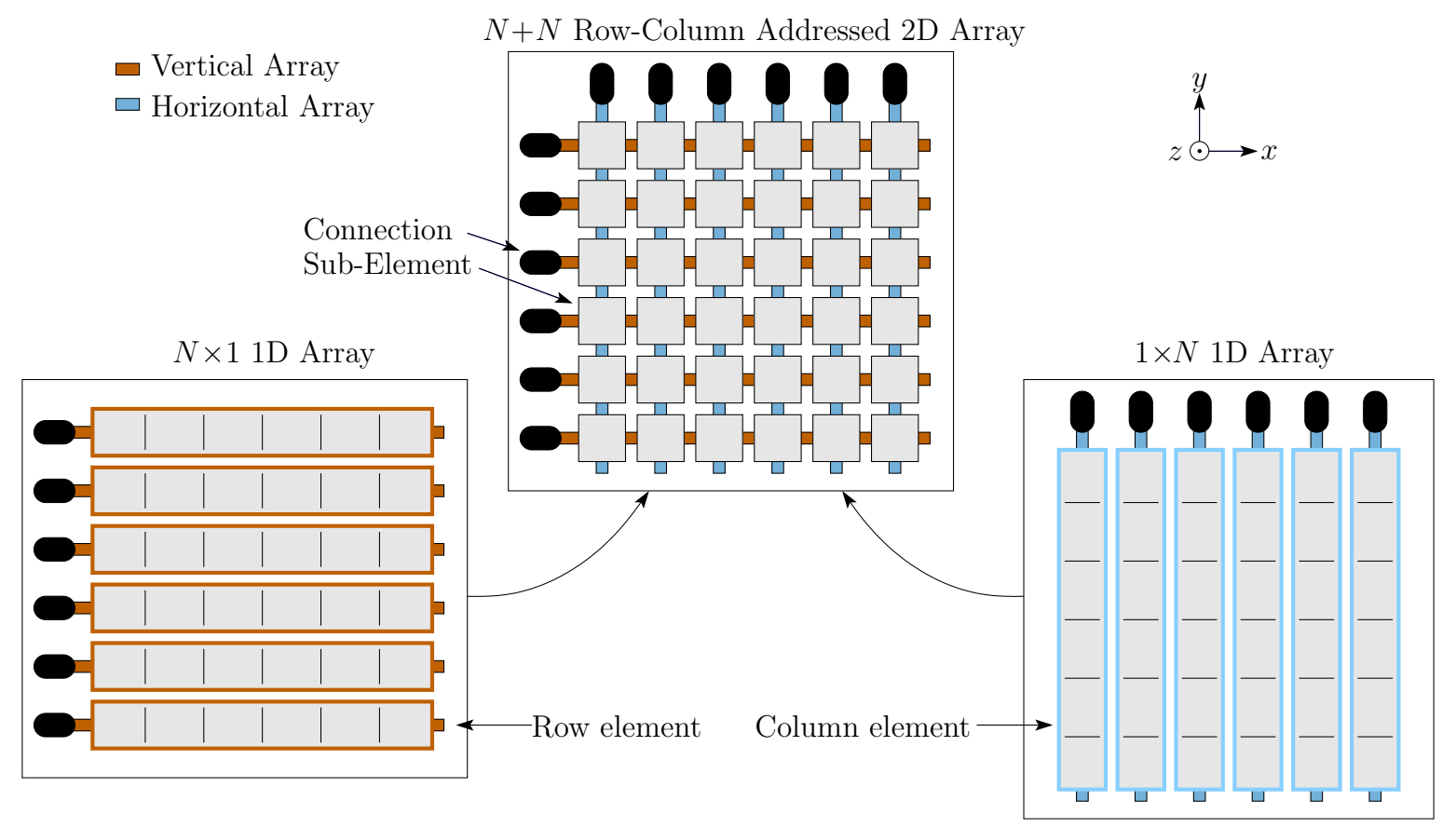

Figure 1: A row-column addressed 2D array can be interpreted as two orthogonal 1D arrays: One array consisting of row-elements and one array consisting of column elements.

a row-column addressed array is shown to give rise to ghost echoes. Apodization of the sub-elements is proposed as a solution to reduce the ghost effects. Finally, the imaging performance of a row-column addressed array is inspected and compared with the imaging quality of a fully addressed 2D array.

\section{METHODS/THEORY}

This section describes how 3D focusing can be achieved with a row-column addressed array, what its disadvantages are, and what can be done to minimize them.

\subsection{Focusing}

Each of the emulated 1D arrays can focus in one dimension. When using the vertical array, seen in Fig. 1, as a transmit array and the horizontal from the same figure as a receive array, the $x$-dimension can be dynamically focused in receive and the $y$-dimension focused in transmit. The $y$-dimension can be dynamically focused in transmit when using synthetic transmit focusing. The emitted wavefront of a single element has the shape of a cylinder surface: it is a plane wave in the $z-x$ plane and a circle arc in $z-y$ plane. This is illustrated in Fig. 2. The time of flight is given by the shortest distance from the source to the scatterer and back to the receiving element, divided by the speed of sound. Using the notation from Fig. 2, this can be written as:

$$
t_{T o F}=\frac{\left\|\mathbf{r}_{\mathbf{e}}\right\|+\left\|\mathbf{r}_{\mathbf{r}}\right\|}{c},
$$

where $\mathbf{r}_{\mathbf{e}}$ is the vector from the emitting element to the focus point, $\mathbf{r}_{\mathbf{r}}$ is the vector from the focus point and back to the receiving element, and $c$ is the speed of sound in the medium. Since the length of each element is large, the usual assumption that the elements are point sources no longer holds. Therefore, $\mathbf{r}_{\mathbf{e}}$ and $\mathbf{r}_{\mathbf{r}}$ connects the focus point to the closest point on respectively the source element and the receiving element. This can also be seen in Fig. 2.

Determining $\left\|\mathbf{r}_{\mathbf{e}}\right\|$ and $\left\|\mathbf{r}_{\mathbf{r}}\right\|$ is solved by calculating the distance between an arbitrary point in 3D, the focus point $\mathbf{P}$, and a line segment $\mathbf{A B}$ from point $\mathbf{A}$ to point $\mathbf{B}$. This is illustrated in Fig. 3 . The projection of the 


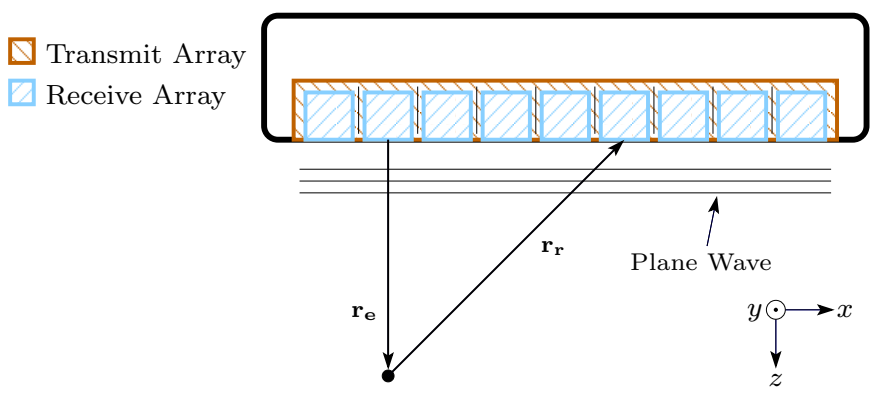

(a) ToF in $x$-dimension.

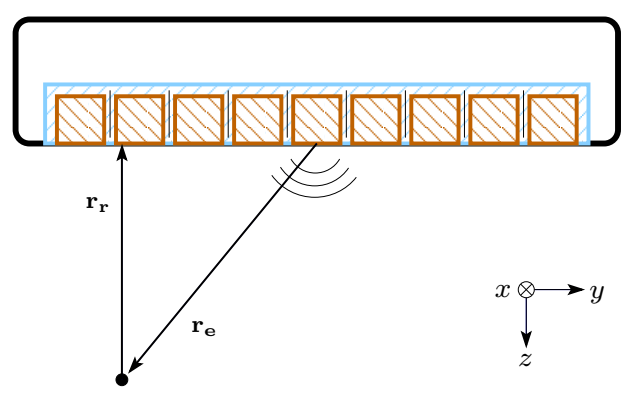

(b) ToF in $y$-dimension.

Figure 2: Time of flight (ToF) illustration of one emission to the focus point and back to one receiving element. $\mathbf{r}_{\mathrm{e}}$ is the vector from the nearest point of the emitting element to the focus point and $\mathbf{r}_{\mathbf{r}}$ is the vector from the focus point to the nearest point on the receive element.

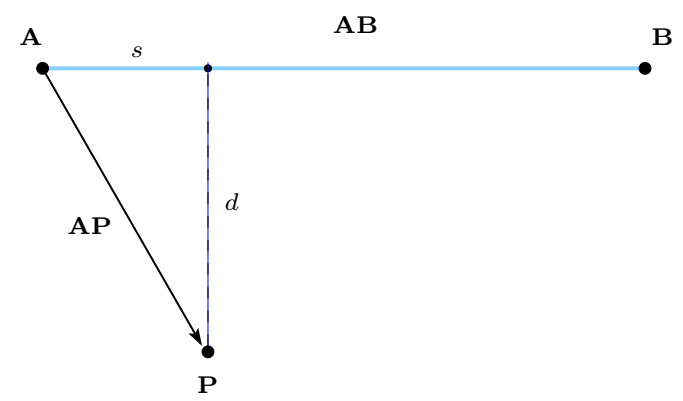

Figure 3: Projection of the point $\mathbf{P}$ onto the line segment AB. $s$ is the distance from $\mathbf{A}$ to the projected point and $d$ is the shortest distance from $\mathbf{P}$ to $\mathbf{A B}$.

point $\mathbf{P}$ onto the line $\mathbf{A B}$ is found using the dot product:

$$
s=\frac{\mathbf{A P} \cdot \mathbf{A B}}{\|\mathbf{A B}\|}
$$

where $s$ is the distance between $\mathbf{A}$ and the projection of $\mathbf{P}$ onto $\mathbf{A B} . s$ is positive if the projected point is located on the same side of $\mathbf{A}$ as $\mathbf{B}$, and negative if it is located on the other side. By normalising $s$ with the length of the line segment it is mapped to lie in the interval $[0,1]$, when the projected point is located on the line segment (between $\mathbf{A}$ and $\mathbf{B}$ ):

$$
\hat{s}=\frac{s}{\|\mathbf{A B}\|}=\frac{\mathbf{A P} \cdot \mathbf{A B}}{\|\mathbf{A B}\|^{2}} .
$$

When the projection of $\mathbf{P}$ onto the line lies between $\mathbf{A}$ and $\mathbf{B}$, i.e. when $\hat{s} \in[0,1]$, the standard formula for the distance between a line and a point can be used:

$$
d=\frac{\|\mathbf{A B} \times \mathbf{A P}\|}{\|\mathbf{A B}\|} .
$$

When $\hat{s} \notin[0,1]$, the shortest distance from the line segment to the point is the distance from either one of ends of the line segment $(\mathbf{A}$ or $\mathbf{B})$ to the point $(\mathbf{P})$. The following therefore determines the minimum distance between 
the point $\mathbf{P}$ and the line segment $\mathbf{A B}$ :

$$
d(\mathbf{A B}, \mathbf{P})= \begin{cases}\frac{\|\mathbf{A B} \times \mathbf{A} \mathbf{P}\|}{\|\mathbf{A B}\|} & \text { if } 0 \leq \hat{s} \leq 1, \\ \|\mathbf{A} \mathbf{P}\| & \text { if } \hat{s}<0, \\ \|\mathbf{B} \mathbf{P}\| & \text { if } \hat{s}>1 .\end{cases}
$$

Using (5), the distances $\left\|\mathbf{r}_{\mathbf{e}}\right\|$ and $\left\|\mathbf{r}_{\mathbf{r}}\right\|$ can now be determined as

$$
\left\|\mathbf{r}_{\mathbf{e}}\right\|=d\left(\mathbf{x m t}_{m}, \mathbf{P}\right) \quad \text { and } \quad\left\|\mathbf{r}_{\mathbf{r}}\right\|=d\left(\mathbf{r c v}_{n}, \mathbf{P}\right)
$$

where $\mathbf{x m t}_{m}$ is the $\mathbf{A}$ and $\mathbf{B}$ coordinates of the $m$ 'th transmit element and $\mathbf{r c v}_{n}$ is the corresponding coordinates for the $n$ 'th receive element. By inserting (6) into (1), we arrive at

$$
t_{T o F_{m, n}}(\mathbf{P})=\frac{d\left(\mathbf{x m t}_{m}, \mathbf{P}\right)+d\left(\mathbf{r c v}_{n}, \mathbf{P}\right)}{c} .
$$

The focused signal at point $\mathbf{P}$ is given by summing all receive signals at the time instances given by (7):

$$
s(\mathbf{P})=\sum_{m=1}^{M} \sum_{n=1}^{N} a_{n} y_{m, n}\left(t_{T o F_{m, n}}(\mathbf{P})\right),
$$

where $M$ is the number of transmit elements, $N$ is the number of receive elements, $a_{n}$ is the receive apodization, $y_{m, n}(t)$ is the received signal from emission $m$ to receive element $n$ at time $t$.

\subsection{Area and resolution scaling}

The row-column addressed array is similar to the Mills cross array, which has been used and analysed by several authors. ${ }^{14,15}$ They both consist of two orthogonal 1D arrays. The difference is that for each channel to the cross array there is one physical element, and for each channel to the row-column array there is an entire row or column of physical elements. The row-column addressed array can therefore emit far more energy than the cross array, or any other of the sparse arrays.

In the following, a comparison of the row-column addressing is made with the sparse 2D Mills cross and the fully sampled 2D array. The Mills cross consists of $2 N-1$ elements on a $N \times N 2 \mathrm{D}$ array. In Fig. 4(a) the active

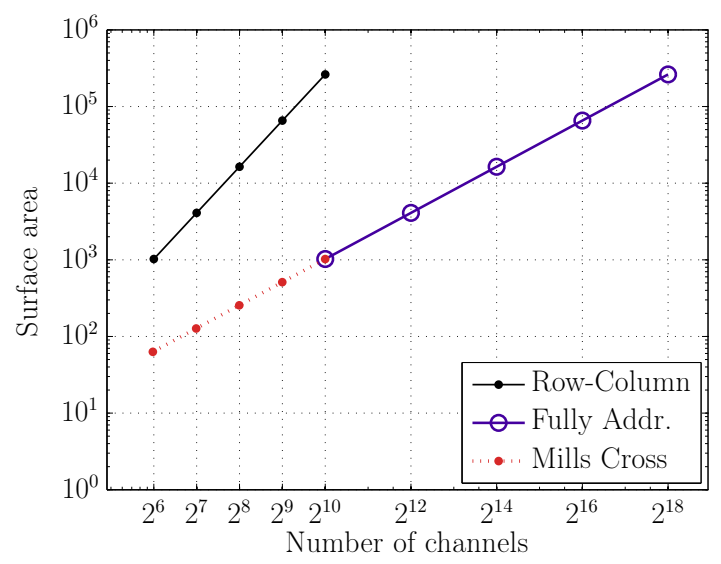

(a) Surface area comparison.

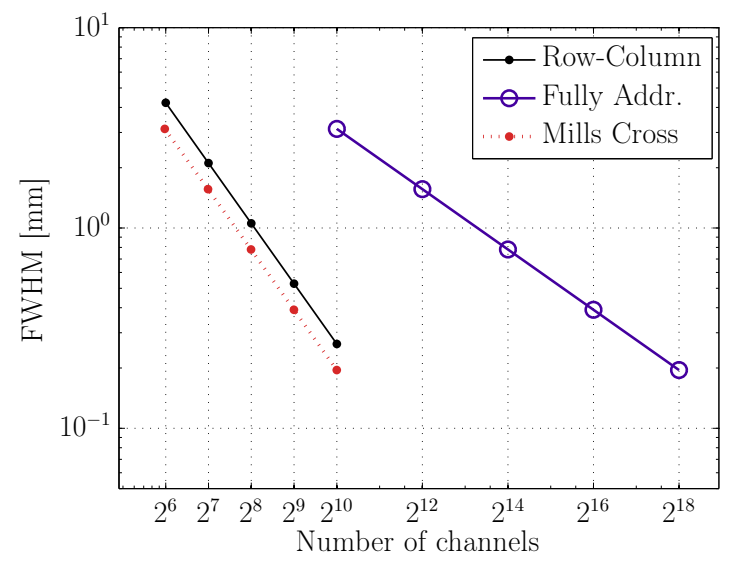

(b) Detail resolution comparison.

Figure 4: Comparison of three transducer layouts. The marked points indicate $32 \times 32,64 \times 64,128 \times 128,256 \times 256$ and $512 \times 512$ element $2 \mathrm{D}$ arrays. 


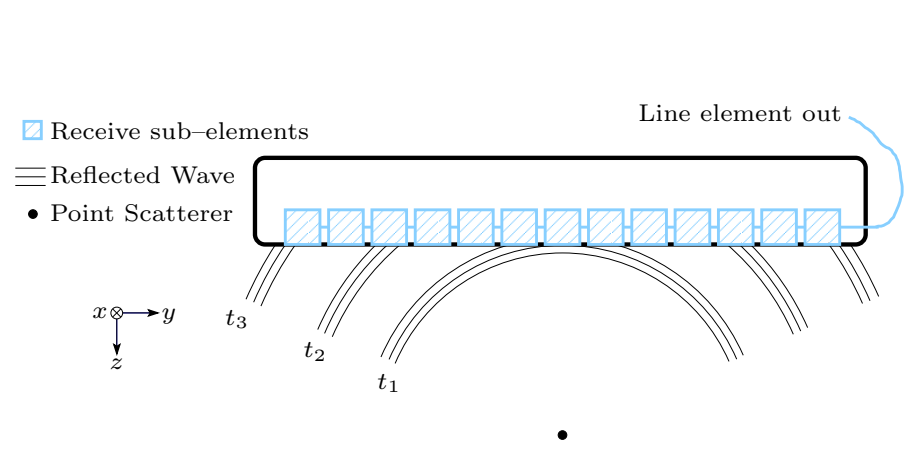

(a) Receiving the reflected wave at the three time instants: $t_{1}$, $t_{2}$ and $t_{3}$.
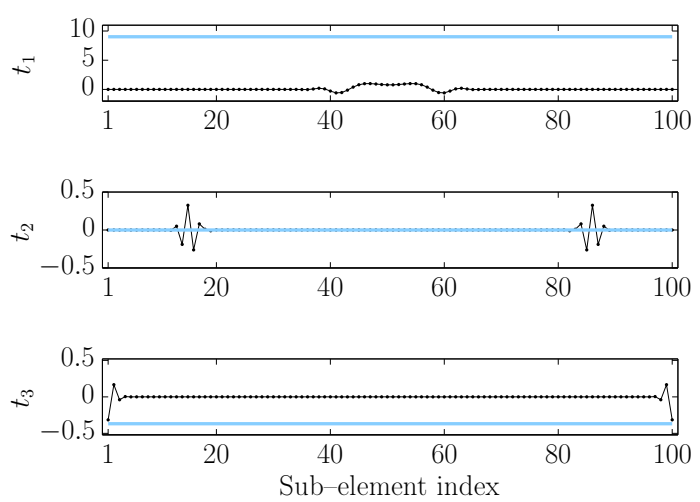

(b) The measured signal. The response of each subelement is shown in black and the output of the line element is shown in light blue.

Figure 5: Edge effect illustration. The three plots in (b) corresponds to the measured signal in (a) at the three time instants $t_{1}, t_{2}$ and $t_{3}$. The amplitudes in (b) are normalized to the maximum of the sub-element signals.

area of the row-column addressed array, the fully addressed array and the Mill Cross is plotted. The marked points indicate five different array sizes: $32 \times 32,64 \times 64,128 \times 128,256 \times 256$ and $512 \times 512$ element $2 \mathrm{D}$ arrays. As expected, the surface area per channel is much larger for the row-column array than for the two other arrays. The row-column addressed array is seen to have one order of magnitude larger surface area than a cross array for a $32 \times 32$ element array. When using $1024\left(2^{10}\right)$ channels, the surface area of the row-column addressed array is more than two orders of a magnitude larger than the surface area of the two other arrays.

In Fig. 4(b) a rough estimate of full width at half maximum (FWHM) for each array is plotted. This prediction is only for the main-lobe size and says nothing about the side-lobe levels. The estimate builds on the standard formula for the main-lobe width:

$$
\mathrm{FWHM}=\lambda F \#=\frac{\lambda d}{w},
$$

where $d$ is the depth to the focal point, $w$ is the width of the array and $\lambda$ is the wavelength. The above equation assumes focusing in both transmit and receive. Since the row-column addressed array only focuses one-way in each dimension, a rough estimate is made that its main-lobe size is $35 \%$ wider than predicted by (9). The row-column addressed array is seen to perform almost as good as the Mills cross and much better than the fully addressed 2D array for the same amount of elements.

That the row-column addressed array both has a large surface area and promises to achieve a high resolution per active channel is what makes it a very interesting candidate for real-time 3D imaging.

\subsection{Edge effects}

When an emitted waveform is reflected by a point scatterer will the reflected wave have the shape of a sphere. Just as the reflected spherical wave intersects with the receiving line element, will only the first part of the wave interact with the sub-elements. This is illustrated as the wave at $t_{1}$ in Fig. 5(a). The measured signal from the line element is a sum of the sub-element signals. At time $t_{1}$ will the signals from the sub-elements be mostly in phase, which is illustrated in the top plot of Fig. 5(b). The line element therefore outputs a strong signal at $t_{1}$. A short time later, the entire reflected wave intersects with the line element at two locations. This is illustrated as the wave at time $t_{2}$. The amplitude of the transmit waveform used in medical ultrasound is usually symmetric around zero and the integration of the waveform is therefore close to zero. As seen in the center plot in Fig. 5(b), the output from the line element at $t_{2}$ is very close to zero. The output of the line element is zero as long as 
Table 1: Simulation parameters

\begin{tabular}{lccl}
\hline \hline Parameter name & Notation & Value & Unit \\
\hline Center frequency & $f_{0}$ & 3.5 & $\mathrm{MHz}^{-1}$ \\
Speed of sound & $c$ & 1540 & $\mathrm{~m} \mathrm{~s}^{-1}$ \\
Wave length & $\lambda$ & 440 & $\mathrm{\mu m}$ \\
Transducer pitch $-x$ & $d_{x}$ & 440 & $\mu \mathrm{m}$ \\
Transducer pitch $-y$ & $d_{y}$ & 440 & $\mathrm{\mu m}$ \\
Sampling frequency & $f_{s}$ & 100 & $\mathrm{MHz}$ \\
Emission pulse & - & 2-cycles, Hann-weighted & - \\
\hline \hline
\end{tabular}

the entire waveform intersects with it. The first part of the waveform does at the time $t_{3}$ not intersect with the line element anymore. The line element therefore has a small output at $t_{3}$, which is shown in the bottom plot in Fig. 5(b).

To minimize this effect, the edge sub-elements should have a lower weighting. This can be achieved by applying an apodization window on the physical sub-elements within the line elements. This apodization is in this work referred to as hardware or sub-element apodization. The hardware apodization is applied in the horizontal direction when using the vertical array in Fig. 1, and vice versa.

\section{SIMULATION SETUP}

All simulations are carried out using Field $\mathrm{II}^{16,17}$ and beamformed using BFT3. ${ }^{18}$ The transducer used in the simulations is a row-column addressed $128+128$ element 2D array. The exception is the fully addressed 2D array, used for comparison in Fig. 8 , which is a $16 \times 16$ 2D array. The simulation parameters can be seen in Table 1.

The point spread function is simulated by imaging a volume containing a single point scatter. The point scatterer is in all simulations placed in front of the transducer at $(x, y, z)=(0,0,50) \mathrm{mm}$. With both transducers a single element is used per emission. This leads to 128 emissions for the row-column addressed array and to 256 emissions for the fully addressed array. All apodizations applied are implemented using a Hann window.

The row-column array is emulated by simulating a fully addressed $128 \times 128$ array and then summing the responses of all elements of each column. This results in one signal per column. In transmit, the same waveform is applied to all elements within the same row. Hence, one waveform is used per row.

\section{RESULTS}

In this section, the results of predicting the time of flight and the effect of using apodization is presented.

\subsection{Time of flight prediction}

The gray scale images in Fig. 6 represent the envelope of the simulated received signal after column element 64 has emitted the transmit waveform. The earliest arrival is the main wavefront, and the following wavefronts appear because of the edge effect. Because of symmetry with the scatterer located at $(x, y)=(0,0)$, only two dominating edge waves appear. The overlaid lines are the calculated arrival times of each wavefront. The lines are seen to be right on top of the wavefront envelopes and they thereby predict their arrival time.

In Fig. 6(a), no hardware apodization is used and two ghost echoes are clearly visible. The maximum amplitude of the two ghost echoes are $-18.7 \mathrm{~dB}$ and $-42.1 \mathrm{~dB}$ relative to the main wavefront. After applying hardware apodization, the two ghost echoes are suppressed to $-40.5 \mathrm{~dB}$ and $-93.0 \mathrm{~dB}$, which is seen in Fig. 6(b). 


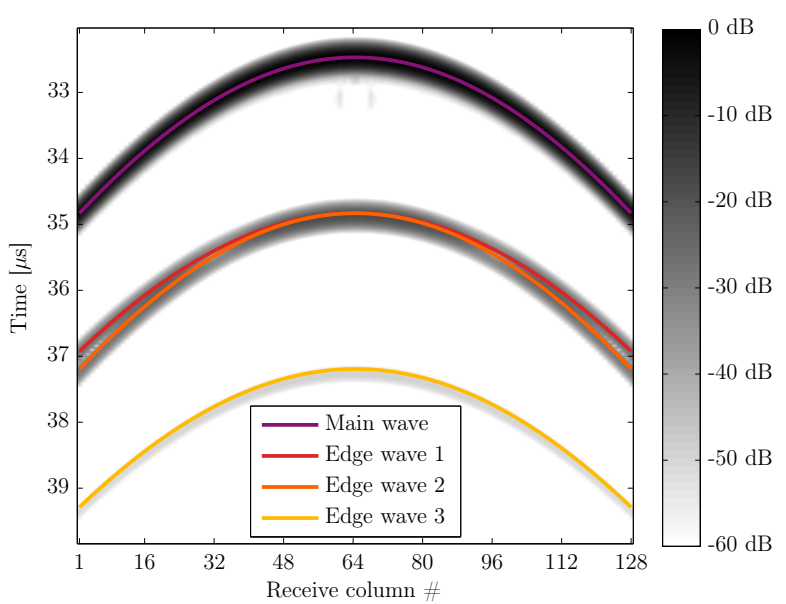

(a) Without apodization.

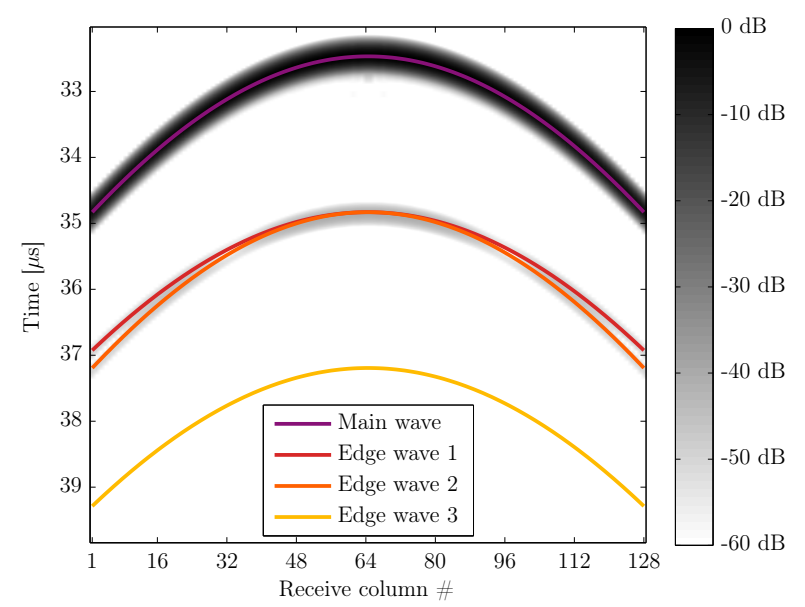

(b) With apodization.

Figure 6: Time of flight for a scattere located at $(x, y, z)=(0,0,50) \mathrm{mm}$. The gray scale image is the logcompressed envelope of the simulated received signal of each column. The overlaid colored lines are the prediction of arrival time of each wave.

\subsection{Apodization effects}

The effect on the PSF of applying apodization is shown in Fig. 7. In Fig. 7(c), traditional apodization is applied during the beamforming. In Fig. 7(b), hardware apodization, described in section 2.3, is applied directly on the sub-elements of each transmit and receive row/column element. No apodization is applied in Fig. 7(a) and in Fig. $7(\mathrm{~d})$ are both apodization types are applied.

The conventional beamforming apodization is seen to greatly reduce the side lobes of both the main response and the ghosts. The cost is a slightly larger main-lobe. The hardware apodization suppresses the ghosts, but has no influence on the main response. The maximum ghost intensity is reduced by the hardware apodization with $22.2 \mathrm{~dB}$ from $-22.3 \mathrm{~dB}$ to $-44.5 \mathrm{~dB}$.

A comparison between the row-column addressed array and a fully addressed array with the same amount of channels is shown in Fig. 8. The PSF of the fully addressed array is seen to have both a wider main-lobe and larger side-lobes than the row-column addressed array. The FWHM in the lateral direction ( $x$-dimension) of the row-column addressed array is $1.01 \mathrm{~mm}$, while it is $2.81 \mathrm{~mm}$ for the fully addressed array. This shows, that when using the same amount of channels in the ultrasound imaging system, but changing the transducer array from a fully addressed array to a row-column addressed array, the resolution can be increased by more than a factor of two. As seen from Fig. 4(b), this factor increases with larger arrays.

The cystic resolution describes the ability to detect an anechoic cyst in a uniform scattering medium. Specifically, the cystic resolution gives the intensity at the center of an anechoic cyst of a given size relative to its surrounding uniformly back-scattering medium. ${ }^{19}$ The lower the intensity at the center of the cyst, i.e. the darker it appears in the image for a given cyst size, the better imaging performance of the system. The relative intensity (RI) of the anechoic cyst was shown by Ranganathan and Walker ${ }^{20}$ to be quantized as the clutter energy to total energy ratio,

$$
\mathrm{RI}(R)=\sqrt{\frac{E_{\text {out }}(R)}{E_{\text {tot }}}}=\sqrt{1-\frac{E_{\text {in }}(R)}{E_{\text {tot }}}},
$$

where $E_{\text {in }}$ is the signal energy inside a circular region with radius, $R$, centered on the peak of the point spread function. $E_{t o t}$ is the total point spread function energy and $E_{\text {out }}$ is the point spread function energy outside the 


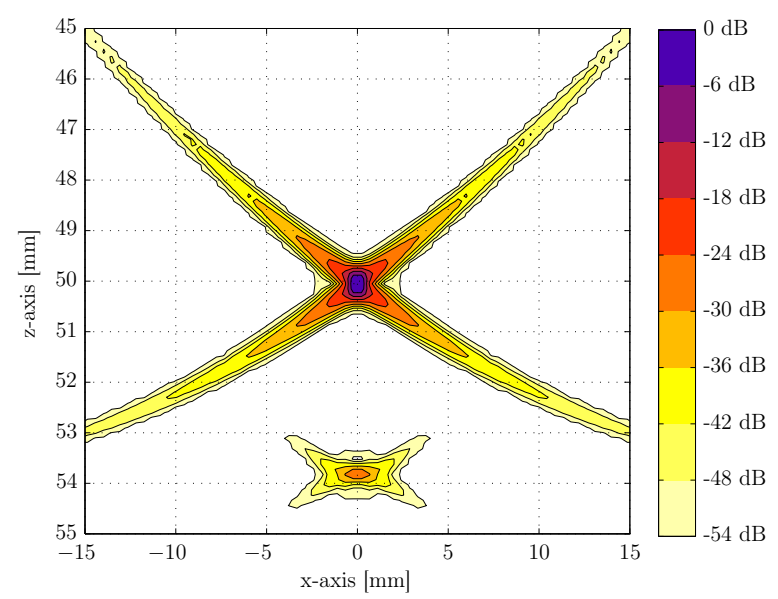

(a) Without apodization.

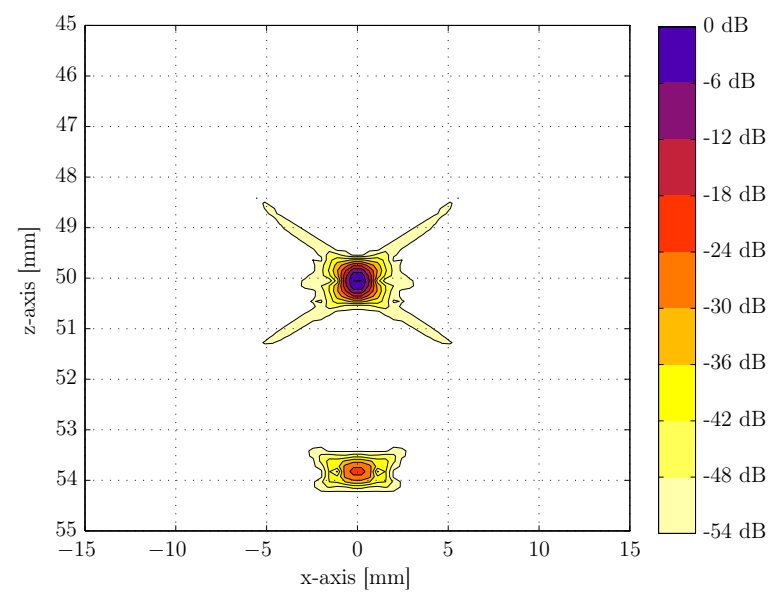

(c) With beamforming apodization.

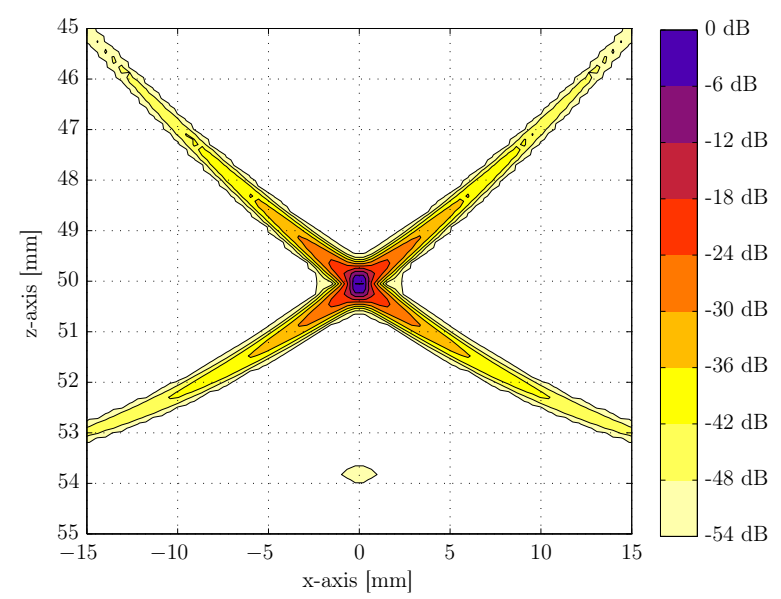

(b) With hardware apodization.

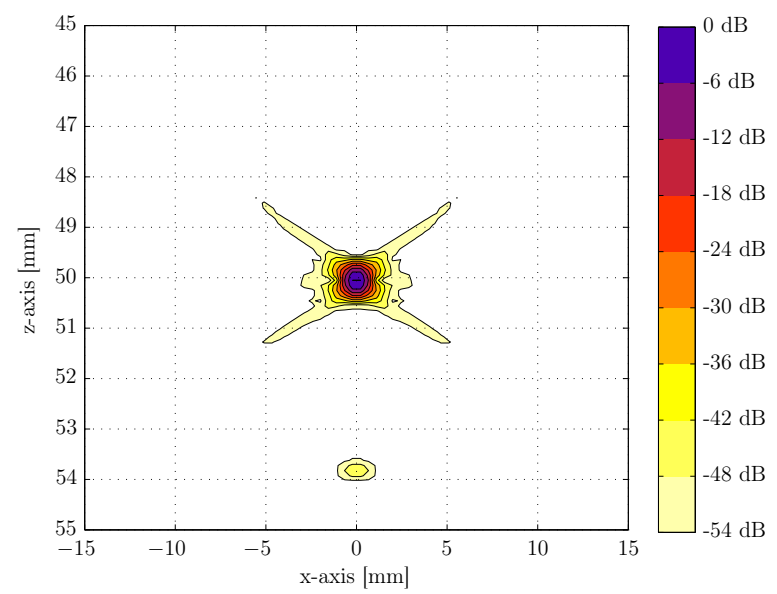

(d) With both beamforming and hardware apodization.

Figure 7: Effect on PSF of using different apodizations. The scatterer is located at $(x, y, z)=(0,0,50) \mathrm{mm}$. The hardware apodization is seen to not affect the main response, but to suppress ghosts. 


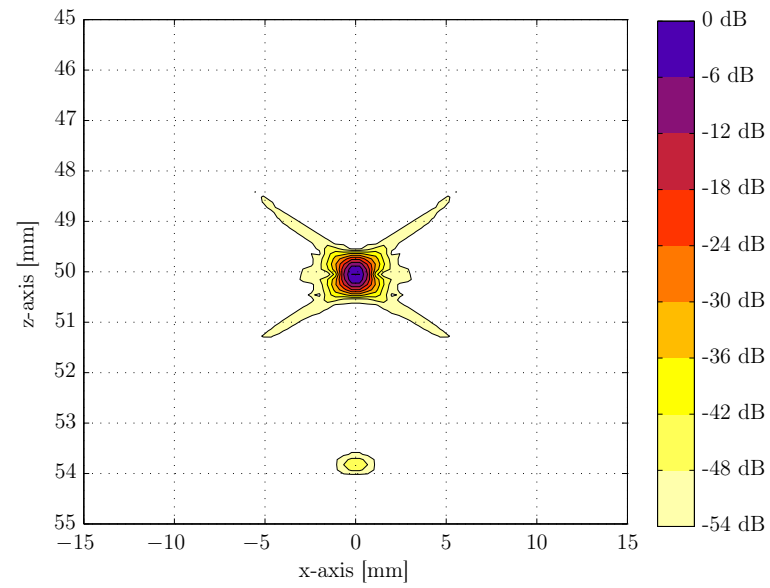

(a) PSF in $z-x$ plane of the row-column addressed array.

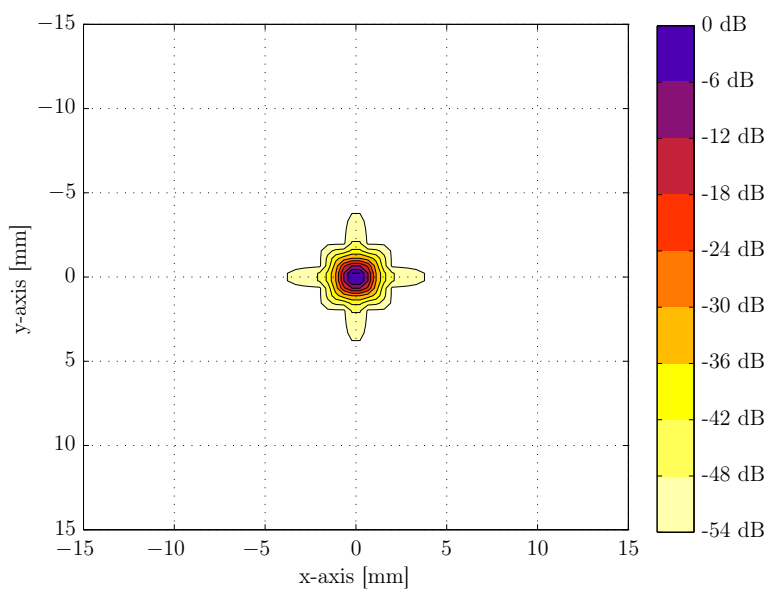

(c) PSF in C-scan at $50 \mathrm{~mm}$ depth of the row-column addressed array.

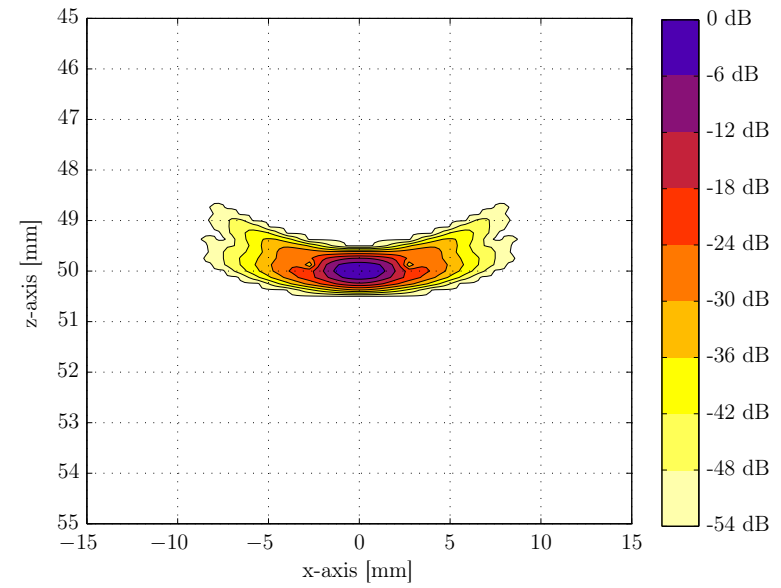

(b) PSF in $z-x$ plane of the fully addressed array.

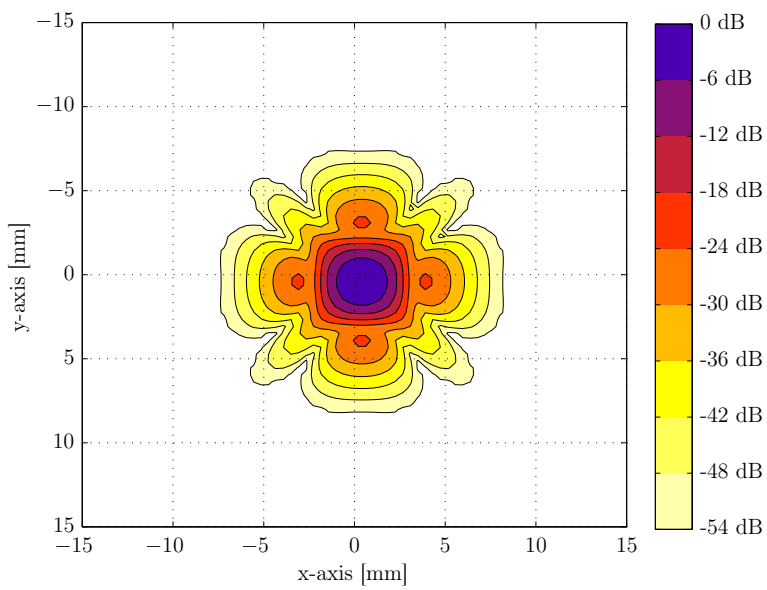

(d) PSF in C-scan at $50 \mathrm{~mm}$ depth of the fully addressed array.

Figure 8: Comparison of the achievable PSF from a row-column addressed array and a fully addressed array, with the same number of active elements. As a result of both the $x$-dimension (in receive) and the $y$-dimension (in transmit) being dynamically focused is the performance in the two dimensions completely identical. 
circular region. To achieve a single number from the $\mathrm{RI}(R)$-curve, the required radius to achieve a $20 \mathrm{~dB}$ relative intensity between the center of the cyst and its surroundings is determined. This is written as $R_{20 \mathrm{~dB}}$.

The $-20 \mathrm{~dB}$ cystic resolution is in the $z-x$ plane $0.76 \mathrm{~mm}$ for the row column-addressed array and $3.16 \mathrm{~mm}$ for the fully addressed array. This indicates that the achievable contrast will be much higher with the row-column addressed array.

\section{CONCLUSION AND PERSPECTIVES}

The imaging quality of a row-column addressed array, that promises to greatly reduce the amount active channels used in 3D and 4D ultrasound imaging, was presented. It was demonstrated how focusing can be achieved with a row-column addressed array. The PSF was shown to contain ghosts, originating from edge effects of the long row and column elements. When using synthetic transmit focusing and applying both beamforming and hardware apodization, the imaging quality of the row-column addressed array was shown to be superior to the imaging quality of a fully addressed array using the same amount of channels. The array simulated uses only 128 channels in transmit and 128 channels in receive to achieve 3D imaging. The array also has a large footprint, which gives it the potential to achieve a high penetration depth and signal-to-noise ratio. The array thereby seems very promising for $3 \mathrm{D}$ and $4 \mathrm{D}$ imaging. Even for 2D imaging, the array could be used. In 2D imaging, it could do plane wave imaging with the out-of-plane dimension dynamically one-way focused.

\section{ACKNOWLEDGMENTS}

This work was financially supported by grant 024-2008-3 from the Danish Advanced Technology Foundation and from BK Medical, Herlev, Denmark.

\section{REFERENCES}

[1] von Ramm, O. T., Smith, S. W., and Pavy, H. G., "High-speed ultrasound volumetric imaging system Part II: Parallel processing and image display," IEEE Trans. Ultrason., Ferroelec., Freq. Contr. 38, 109-115 (1991).

[2] Turnbull, D. and Foster, F., "Beam steering with pulsed two-dimensional transducer arrays," IEEE Trans. Ultrason., Ferroelec., Freq. Contr. 38, 320-333 (July 1991).

[3] Rasmussen, M. F., Hansen, J. M., Férin, G., Dufait, R., and Jensen, J. A., "Preliminary comparison of 3D synthetic aperture imaging with Explososcan," in [Proc. SPIE Med. Imag.], Bosch, J. G. and Doyley, M. M., eds., 8320(1), 83200F 1-9, SPIE (2012).

[4] Rasmussen, M. F., Férin, G., Dufait, R., and Jensen, J. A., "Comparison of 3D synthetic aperture imaging and explososcan using phantom measurements," in [Proc. IEEE Ultrason. Symp.], Accepted (2012).

[5] Davidsen, R. E., Jensen, J. A., and Smith, S. W., "Two-dimensional random arrays for real time volumetric imaging," Ultrasonic Imaging 16, 143-163 (July 1994).

[6] Brunke, S. S. and Lockwood, G. R., "Broad-bandwidth radiation patterns of sparse two-dimensional vernier arrays," IEEE Trans. Ultrason., Ferroelec., Freq. Contr. 44, 1101-1109 (sep 1997).

[7] Yen, J., Steinberg, J., and Smith, S., "Sparse 2-D array design for real time rectilinear volumetric imaging," IEEE Trans. Ultrason., Ferroelec., Freq. Contr. 47, 93-110 (January 2000).

[8] Austeng, A. and Holm, S., "Sparse 2-D arrays for 3-D phased array imaging - design methods," IEEE Trans. Ultrason., Ferroelec., Freq. Contr. 49, 1073-1086 (August 2002).

[9] Karaman, M., Wygant, I., Oralkan, O., and Khuri-Yakub, B., "Minimally redundant 2-D array designs for 3-D medical ultrasound imaging," IEEE Trans. Med. Imag. 7, 1051-1061 (jul 2009).

[10] Logan, A. S., Wong, L. L., and Yeow, J. T. W., "2-D CMUT wafer bonded imaging arrays with a row-column addressing scheme," in [Proc. IEEE Ultrason. Symp.], 984-987 (sep 2009).

[11] Seo, C. H. and Yen, J. T., "A 256 x 256 2-D array transducer with row-column addressing for 3-D rectilinear imaging," IEEE Trans. Ultrason., Ferroelec., Freq. Contr. 56, 837-847 (apr 2009).

[12] Logan, A. S., Wong, L. L. P., Chen, A. I. H., and Yeow, J. T. W., "A 32 x 32 element row-column addressed capacitive micromachined ultrasonic transducer," IEEE Trans. Ultrason., Ferroelec., Freq. Contr. 58, 12661271 (jun 2011). 
[13] Chen, A. I. H., Wong, L. L., Logan, A. S., and Yeow, J. T. W., "A CMUT-based real-time volumetric ultrasound imaging system with row-column addressing," in [Proc. IEEE Ultrason. Symp.], 1755-1758 (oct 2011).

[14] Smith, S. W., Pavy, H. G., and von Ramm, O. T., "High-speed ultrasound volumetric imaging system Part I: Transducer design and beam steering," IEEE Trans. Ultrason., Ferroelec., Freq. Contr. 38, 100-108 (1991).

[15] Kim, K. and Song, T., "High volume rate 3-D ultrasound imaging using cross array based on synthetic transmit focusing," in [Proc. IEEE Ultrason. Symp.], 2, 1409-1412 (aug 2004).

[16] Jensen, J. A. and Svendsen, N. B., "Calculation of Pressure Fields from Arbitrarily Shaped, Apodized, and Excited Ultrasound Transducers," IEEE Trans. Ultrason., Ferroelec., Freq. Contr. 39, 262-267 (1992).

[17] Jensen, J. A., "Field: A program for simulating ultrasound systems," Med. Biol. Eng. Comp. 10th NordicBaltic Conference on Biomedical Imaging, Vol. 4, Supplement 1, Part 1, 351-353 (1996).

[18] Hansen, J. M., Hemmsen, M. C., and Jensen, J. A., "An object-oriented multi-threaded software beamformation toolbox," in [Proc. SPIE Med. Imag.], 7968, 79680Y 1-9 (March 2011).

[19] Vilkomerson, D., Greenleaf, J., and Dutt, V., "Towards a Resolution Metric for Medical Ultrasound Imaging," in [Proc. IEEE Ultrason. Symp.], 1405-1410 (1995).

[20] Ranganathan, K. and Walker, W. F., "Cystic Resolution: A Performance Metric for Ultrasound Imaging Systems," IEEE Trans. Ultrason., Ferroelec., Freq. Contr. 54(4), 782-792 (2007). 